Classification

Physics Abstracts

$06.50-02.70-07.80$

\title{
Application of multivariate statistical analysis to time dependent spectroscopy
}

\author{
Noël Bonnet $\left({ }^{1}\right)$, Emilia Simova $\left({ }^{2}\right)$ and Xavier Thomas $\left({ }^{1,3}\right)$
}

( $\left.{ }^{1}\right)$ Unité INSERM 314, Université de Reims, 21 rue Clément Ader, 51100 Reims, France

$\left({ }^{2}\right)$ Higher Institute Mechanical Engineering, Center of Applied Mathematics, SOFIA 116, Bulgarie

${ }^{3}$ ) LASSI, Université de Reims, BP 347, 51062 Reims Cedex, France

(Received January 02, 1991; accepted March 05, 1991)

\begin{abstract}
Résumé. - La spectroscopie résolue dans le temps est une méthode prometteuse pour l'étude des événements dynamiques par microanalyse. Dans la mesure où de grandes quantités de données sont produites, des procédures spécialisées d'analyse des données doivent être développées. L'Analyse Statistique Multivariée apparait comme un bon candidat car elle permet une analyse des données sans nécessité d'introduire un modèle d'évolution $a$ priori. Elle est aussi capable d'effectuer un traitement des données (filtrage du bruit par exemple) et une extrapolation des données (extrapolation à dose nulle par exemple). Quelques potentialités de cette méthode sont discutées à partir de plusieurs simulations et à partir de données experimentales dans le domaine de la spectroscopie de rayons $\mathrm{X}$.
\end{abstract}

\begin{abstract}
Time dependent spectroscopy is a promising method for the study of dynamic events by microanalysis. Since it produces a large amount of data, specialized data analysis procedures must be developed. Multivariate Statistical Analysis appears to be a good candidate since it allows data analysis without any a priori model of the evolution. It is also able to perform data processing (noise filtering for instance) and data extrapolation, such as the extrapolation to zero-dose. Some potential uses of this method are explored in several simulations as well as in an experimental example in the field of EDX spectroscopy.
\end{abstract}

\section{Introduction.}

Recent advances in technology allow the development of new acquisition procedures in the field of spectroscopy. One of these recently appearing protocols is time dependent spectroscopy, i.e. the possibility to record series of spectra as a function of time [1-3].

This acquisition procedure (also called chrono-spectroscopy [4]) is useful for studying the evolution of a given element.

In order to be fully exploitable, such new acquisition protocols must be accompanied by new data processing techniques. In particular, the amount of data available becomes so large that conventional methods used routinely in spectroscopy, such as examination and processing of in- 
dividual spectra, cannot be used efficiently. More sophisticated procedures must be devised in order to automatically extract the pertinent information contained in the whole data set.

Among the procedures which can be suggested for that purpose, Multivariate Statistical Analysis (MSA) seems to be a good candidate. The next section presents an overview of the non multiparameter methods which can be used. Then, we recall the principle and possibilities of MSA and finally, we illustrate these potentialities by some simulations and one application to an experimental set.

\section{Overview of some multivariate data processing methods.}

Time dependent spectroscopy provides data (signal intensitiy $I$ ) in a two-dimensional space: energy $E$ (or wavelength $\lambda$ ) and time $t$.

As an example of such a data set, we will use a series of 256 spectra of glass obtained in energy dispersive X-ray (EDX) spectroscopy [2]. These spectra were recorded with a Si-Li detector. Each spectrum is composed of 256 channels and is recorded within 25 seconds.

For the purpose of some subsequent processings, (and due to some present limits of the software), we condensed the original data set into a series of 23 spectra covering the whole acquisition time.

Several non multiparametric representations of the data set can be envisaged:

1) $I(E)$ for a given $t_{0}:$ this is the classical spectroscopic representation. Standard processings can be applied: detection of presence of a given characteristic edge, background fitting, digital derivation, fitting to reference spectra... Figure 1a displays the 23 condensed spectra of the experimental set.
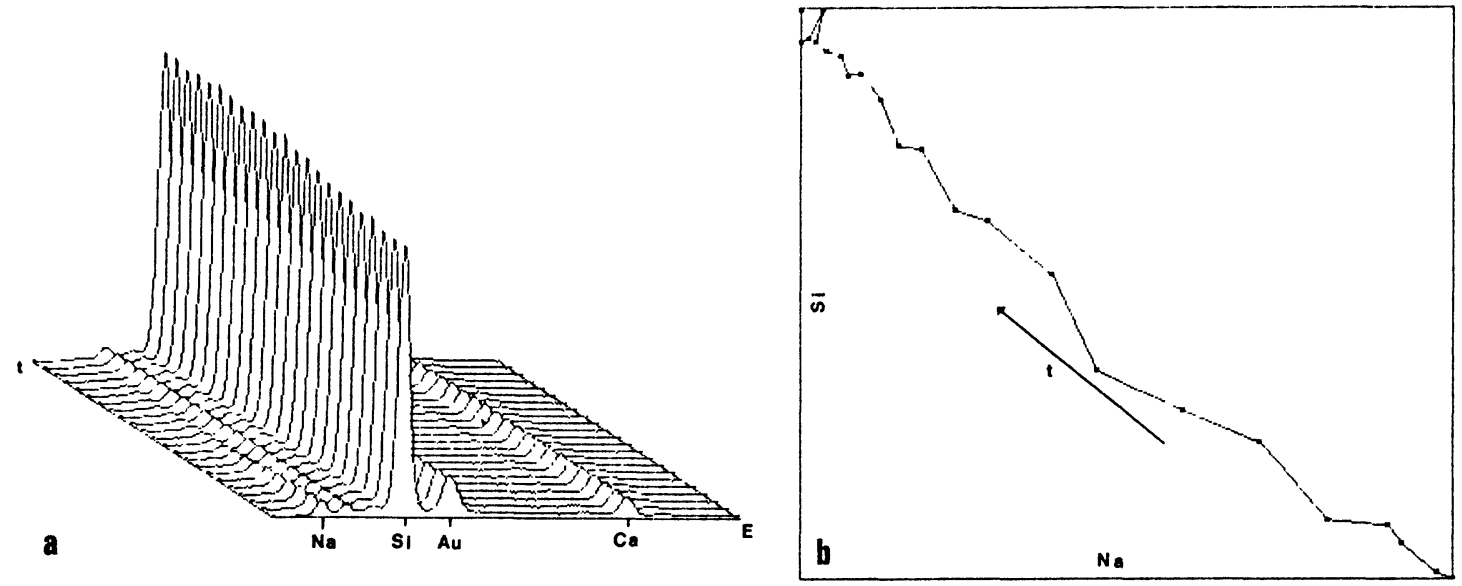

Fig. 1. - a) Series of EDX spectra of glass as a function of time. The original series of 256 spectra was condensed into 23 spectra. b) Scatter diagram showing the time dependence of one element ( $\mathrm{Si}$ ) as a function of another one $(\mathrm{Na})$. We have an apparent anticorrelation between the two evolutions.

2) $I(t)$ for a given $E_{0}$ (or a given energy window $\Delta E$ around $\left.E_{0}\right):$ this gives the evolution of a given elemental content as a function of time. This representation can fulfill the goal of the study when the useful energy channels are known a priori. 
However, this can be insufficient in many situations and can also be dangerous as spurious effects can be introduced by the special acquisition procedure. For instance, part of the evolution of $I(t)$ can be due to a variation in the incident current $I_{0}(t)$.

3) Two-dimensional histogram (or correlation diagram, or Scatter diagram).

The plot of $I(t)$, for a given energy window $\Delta E_{1}$ versus $I(t)$ for another energy window is typically a bivariate representation.

It has been suggested and used by several authors [5-7], in order to visualize and compare the joint evolution of two signals. It is not free of the drawbacks mentioned in the previous paragraph. Figure $1 \mathrm{~b}$ displays the evolution of $\mathrm{Si}$ versus the evolution of $\mathrm{Na}$ in the experimental set.

4) "Picture" representation:

Since the experimental data are in a two-dimensional space, they can be represented as a "picture" where one dimension is $E$ and the other is $t$, the "pixel" content being the signal intensity $I$. Therefore, many image processing tools can be applied to this special image: smoothing, contrast enhancement, background subtraction... Though these procedures could also be applied to each individual spectrum, their application to two-dimensional $(E, t)$ data makes a more efficient use of the global information. An example of an $(E, t)$ image is shown in figures $2 \mathrm{a}, \mathrm{b}$ and the result of image processings (smoothing and enhancement) are shown in figures $2 \mathrm{c}, \mathrm{d}$.

\section{Multivariate statistical analysis.}

Multivariate Statistical Analysis (MSA) has been developed by statisticians $[8,9]$ in order to extract the significant information from large data sets. It became of more practical interest with the advent of computers $[10,11]$. After having been applied to double entry tables (individuals/variables), it has been used for image sequences, which are obviously large data sets in which image numbers play the role of individuals while pixels play the role of variables. Applications have been found in the fields of teledetection, nuclear medicine $[12,13]$, electron microscopy of macromolecules [14-16], Auger microanalysis [17, 18] and electron energy loss microanalysis [19-22].

We will concentrate on some possible applications in spectroscopy, with particular emphasis on time dependent spectroscopy.

3.1 PRINCIPLES AND POTENTIAL USES OF MSA. - The mathematical background of MSA is described in most of the papers mentioned in the previous paragraph and therefore will not be reproduced here. We will only recall the different steps which constitute such an analysis (the notations are the same as in [21]).

- Starting with a data matrix $X=X_{i j}$, where $i$ describes the variables and $j$ the individuals, the overall hypothesis is that the information contained in the data set can be described by the variance-covariance matrix

$$
S=Y^{\mathbf{t}} \cdot Y
$$

where $Y$ is either the data matrix, $X$ itself or a modification of it (normalization, centering...). The different possibilities for this modification define different variants of MSA (Principal Component Analysis, Correspondence Analysis...) [23].

- The analysis of the information contained in the data set can be performed through the diagonalization of the variance-covariance matrix $S$. This is done in order to decompose the global information into orthogonal sources of information. The eigenvectors $v_{\alpha}$ define these sources of information while the associated eigenvalues $\lambda_{\alpha}$ define their relative importance. (The vectors $\alpha$ are ordered by decreasing values of $\lambda_{\alpha}$ ). 


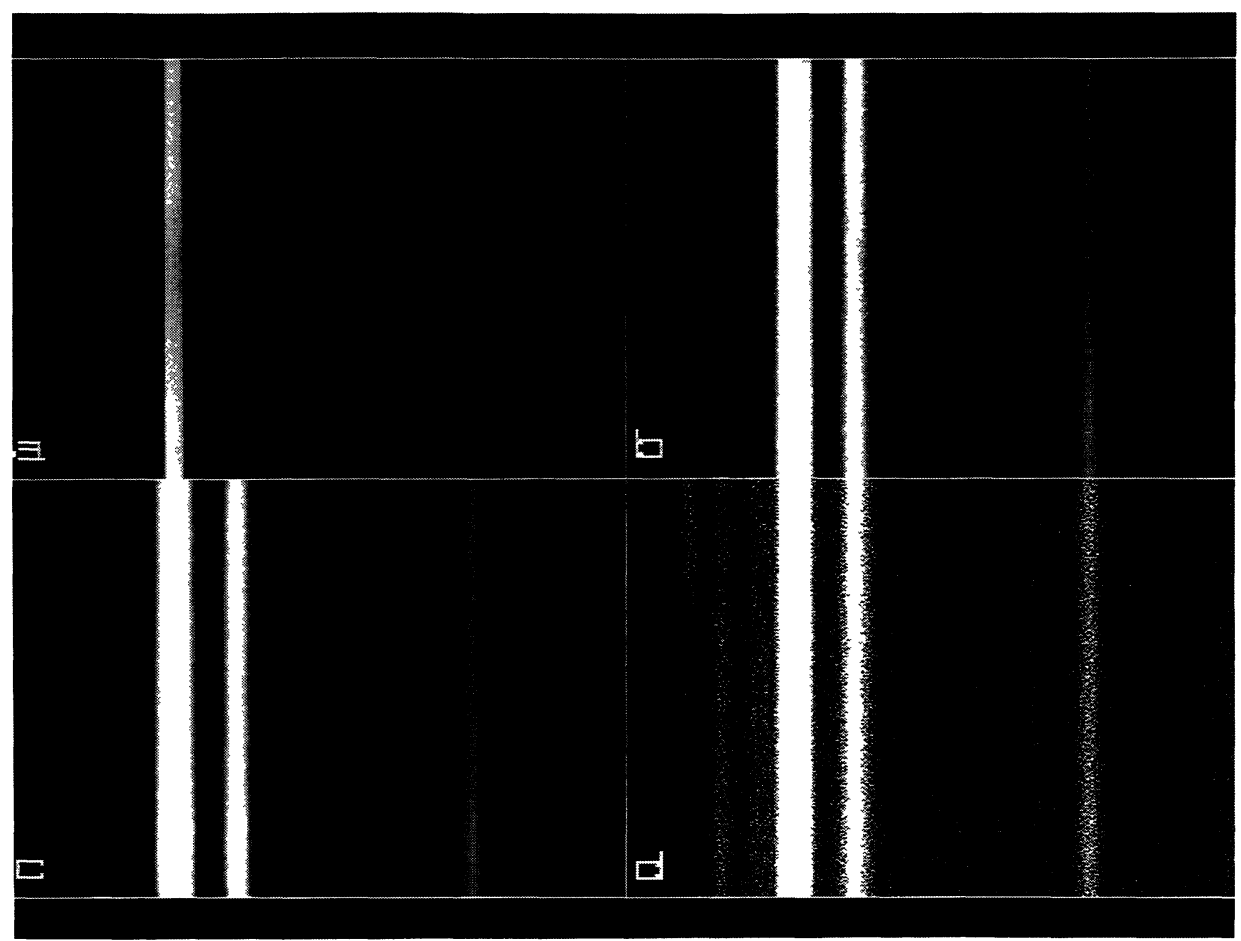

Fig. 2. - a) Representation of the spectrum series as an "image". The horizontal axis represents the energy channels while the vertical axis represents the time. 256 spectra with 256 energy channels are displayed. b) Due to the large dynamical range in these spectra, an upper limit was set to the intensity, resulting in a saturation of the "pixels" in the Si and Au peaks. c, d) Image processing methods were applied to the two-dimensional representation b). c) corresponds to a smoothing by convolutioon over a $3 \times 3$ pixels environment. This smoothing, which results in an improvement of the signal-to -noise ratio, differs from the conventional smoothing applied in spectroscopy, which is an unidimensional smoothing, operating on energy channels only. Here, we also make use of the information contained in several successive spectra, that is, time redundancy. d) corresponds to the reverse operation, i.e. image enhancement, in order to point out details of the phenomena which could not be visualized in the original data. Of course, Poisson noise is also increased.

Once the eigenvalue decomposition is done, the information content can be interpreted by using two tools:

- the visualization of the coordinates of the individuals on the different factorial axes (or eigenvectors): two spectra, close together in the space of the factorial axes carry similar information.

- the computation of the coordinates of the variables (pixels in imagery, energy channels in spectroscopy) on the different factorial axes. These weights of energy channels can be visualized as pseudo-spectra, or spectra of importance, following the terminology "images of importance".

In the case of time dependent spectroscopy, this representation displays the information relative to "which channels carry useful (i.e. non redoundant) information through the spectrum series" and "which energy windows are correlated or anti-correlated with respect to the time evolution".

- Besides the analysis of the data set, MSA also allows some processing of it. One of the processes which can be applied is filtering. The kind of filtering which can be performed by MSA is similar in its philosophy to the frequency filtering which can be applied to individual spectra or 
images, but is also somewhat different. Since MSA splits the whole information into different orthogonal sources of information (eigenvectors), the filtering process consists in keeping some of these sources while discarding others [24]. The spectrum series can then be reconstituted with the eigenvectors which have been retained. For instance, if the original data are noisy, a large part of the noise will be rejected in high order component eigenvectors and can then be discarded in the reconstitution. MSA is thus able to perform a multivariable improvement of the signal-to-noise ratio, which promises to be more valuable than filtering only one spectrum at once.

- Reconstitution of fictitious spectra.

One possibility of MSA which has been developed by one of us (N.B.) is to perform image (or spectrum, or data) interpolation and extrapolation from an experimental image (or spectrum, or data) series. The method is a generalization of the reconstitution described in the previous paragraph. Such reconstitution is performed by combining the variable weights $P_{\alpha i}$ with the individual weights $Q_{\alpha j}$ (see formula 38 in [21]).

The idea for a fictitious reconstitution is to combine the variable weights $P_{\alpha i}$ with "fictitious" individual weights $Q_{\alpha j}$. . Where do these weights come from? They are deduced from the "true" individual weights $Q_{\alpha j}$ following some interpolation or extrapolation. The advantages of performing this interpolation (or extrapolation) in the space of the individual weights, rather than in the original space ( $E, t$, in this specific case), are discussed in a forthcoming paper and will not be detailed here.

One possible application in the field of time dependent spectroscopy is the extrapolation to zero-dose. For diffusible elements, a spectrum series gives some information concerning the evolution of the content in a given element. If one is interested in trying to deduce the content in this element before beginning the experiment, one can try to deduce some parameter (for instance, elemental concentration) from each spectrum of the series and then extrapolate this parameter to zero-dose, following some more or less empirical hypothesis concerning the way to extrapolate.

Another way to perform this extrapolation is to build the "fictitious" spectrum which would result from an experiment at zero-dose. This can be done by the extrapolation procedure described above [25]. It must be stressed that it is still necessary to make more or less realistic hypotheses concerning the physical events which take place at the very beginning of the experiment, but that this extrapolation procedure has the advantage of taking into account the whole information content in the experimental data set.

\section{Demonstration of the applicability of MSA to time resolved spectroscopy (simulations).}

We have performed four simulations in order to exemplify the possibilites described in the previous section. The simulated spectra are composed of one or two characteristic Gaussian peaks, superimposed on a rapidly decreasing background. They look like electron energy loss spectra, but the potentialities of MSA described here are also applicable to any kind of spectroscopy (WDS, EDS, AES...).

4.1 Simulation 1. - In this simulation, two characteristic peaks are superimposed over an $A \cdot E^{-R}$ background. These two peaks have a different evolution as a function of time. The amplitude of the first one (at lower energy) decreases continuously from spectrum $n=1$ to spectrum $n=12$ according to the law $\mathrm{e}^{-0.01 n}$. The amplitude of the other one (at higher energy) first increases linearly from spectrum 1 to spectrum 5 and then decreases linearly from spectrum 6 to spectrum 12. This model of evolution is rather unrealistic but the goal of this simulation is only to show that MSA is able to depict very complicated evolutions. The original spectra are displayed in figure $3 \mathrm{a}$. Figure $3 \mathrm{~b}$ is a representation of the weights of the 12 spectra on axes 1 and 2 , which 
carry $81 \%$ and $19 \%$ of the information, respectively. One can see that the two regimes (1 to 5, 6 to 12) are clearly depicted with this representation. Figure $3 \mathrm{c}$ displays the weights of the different 256 energy channels on axes 1 and 2 . In combination with the previous representation, this one helps in interpreting the data. It tells the user which energy channels contribute to the useful information content (here the channels of the peaks have an important contribution, while the channels of the background carry only redoundant information, which is therefore eliminated by MSA). The interpretation of figure $3 \mathrm{c}$ is that in the evolution of energy channels around numbers 80 and 160, some parts are correlated (6 to 12) while some parts are anti-correlated (1 to 5), thus giving rise to 2 orthogonal information sources (axes 1 and 2). Some further developments of this example would be possible, but would necessitate theoretical considerations which have not been described here, such as oblique analysis.

4.2 Simulation 2. - Here, we have again two Gaussian peaks. The first one decreases as in simulation 1 . The second one has a constant amplitude. However, we simulated a continuous increase of the incident current:

$$
I_{0}(n)=1+0.02 n
$$

Therefore, a bivariate plot of the content in element B as a function of the content in element $\mathrm{A}$ would result in an apparent anticorrelation between the evolution of elements A and B (Fig. 4b).

The projection of the energy channels on axis 1 , which represents $100 \%$ of the information in that case, is displayed in figure $4 \mathrm{c}$ and one can see that only the channels around number 80 carry some significant information. The increase of the intensity in channels around 160 is proportional to the global increase of intensity in the whole spectrum and therefore is not significant.

4.3 Simulation 3. - The goal of this simulation is to investigate the possibilities of depicting small shifts of the characteristic edges which could result from modifications of the chemical bound of the studied atom species as a function of time.

For this study, two characteristics peaks of shape $\mathrm{e}^{-\alpha i}$ (where $i$ is the energy channel) are added to a background. The edge position of the first peak is maintained constant for the five first spectra and then increased linearly (one energy channel shift by spectrum). The edge position of the second peak is kept constant throughout the whole series. Figure 5b shows the result of projecting the energy channels on axis 1 after MSA. One can see that the peak which suffers some drift is clearly identified.

In order to show that this chemical drift cannot be confused with an instrumental drift (due to some high voltage fluctuations for instance), we simulated such a situation. The result is shown in figure 5c. One can see that, in this case, all the characteristic peaks present in the energy region have an important weight on axis 1 , contrary to what was observed in the previous situation.

4.4 EXTRAPOLATION TO ZERO DOSE. - To illustrate this specific point, we simulated a series of spectra containing a characteristic Gaussian peak, superimposed over a decreasing background. The amplitude of this peak is supposed to decrease according to a law: $\mathrm{e}^{-0.2 n}$, where $n$ is the spectrum number (ranging from $n=1$ to $n=6$ ).

In order to obtain a more realistic simulation, we supposed that these spectrum were acquired in a short time and thus, we also simulated Poisson noise. The six simulated spectra are displayed in figure 6a. The weights of the different spectra on axis 1, which represents $47 \%$ of the total variance, are displayed on figure $6 \mathrm{~b}$. (The weights on axis 2 are not displayed, since it can be shown that this factorial axis does not contain anything else than noise). The extrapolation to zero dose cannot be performed without a hypothesis concerning the continuity of the phenomena which happen during $t=0(n=0)$ and $\Delta t(n=1)$. But this requirement is shared by all attempts 

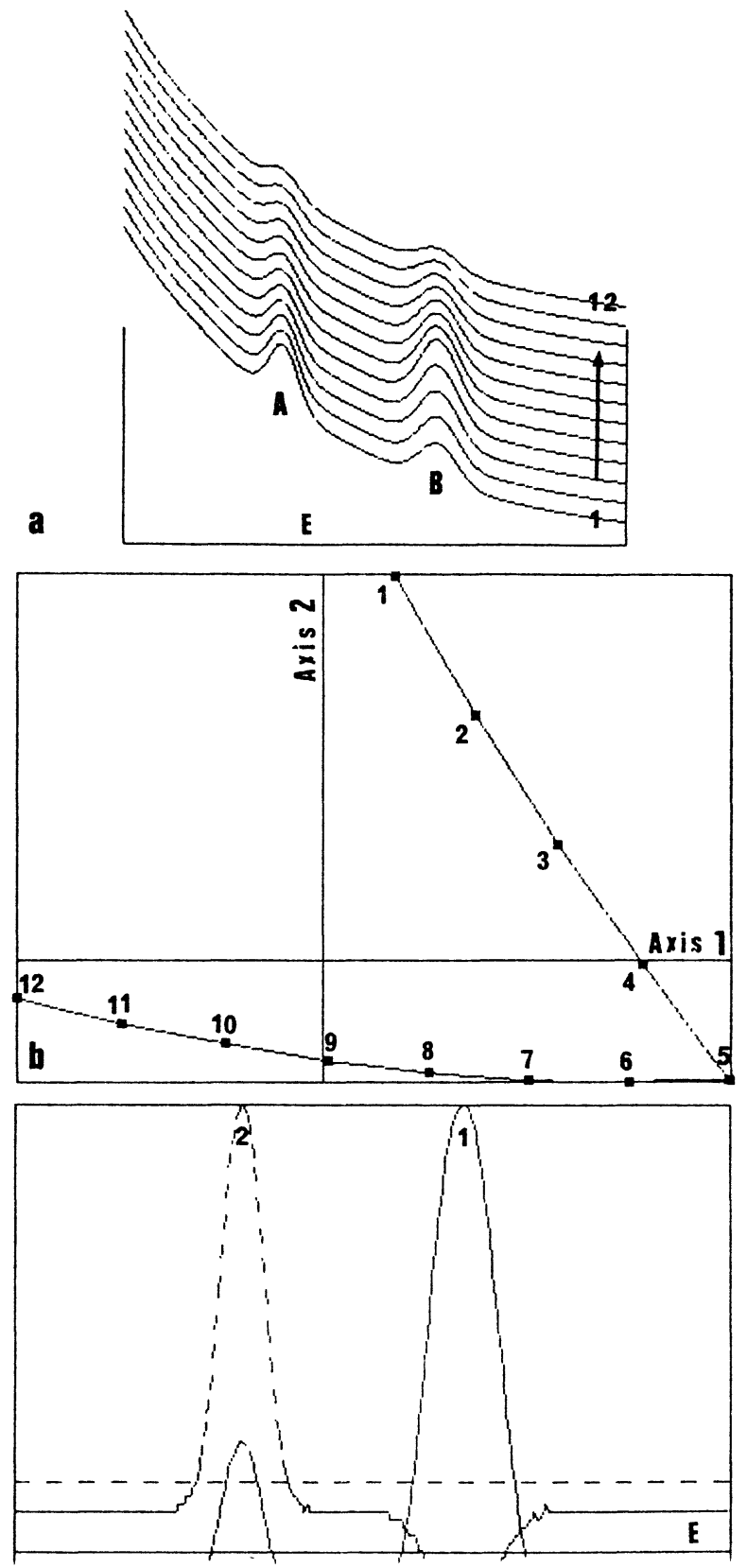

Fig. 3. - a) Time series of 12 simulated spectra. In these spectra, the content of element $A$ is supposed to decrease continuously while the content of element B is supposed to increase first and then decrease (from spectrum 6 to spectrum 12). b) The two regimes are clearly depicted on a plot of the individual spectrum weights on the factorial axes 1 and 2 which result from the Multivariate Statistical Analysis of the data set. c) The projection of the energy channels on the same factorial axes shows which energy channels contain the useful information and explains the meaning of the two factorial axes. Axis 1 describes the correlated evolution of the two elements $\mathrm{A}$ and $\mathrm{B}$, while axis 2 describes the anticorrelated part of this evolution. 

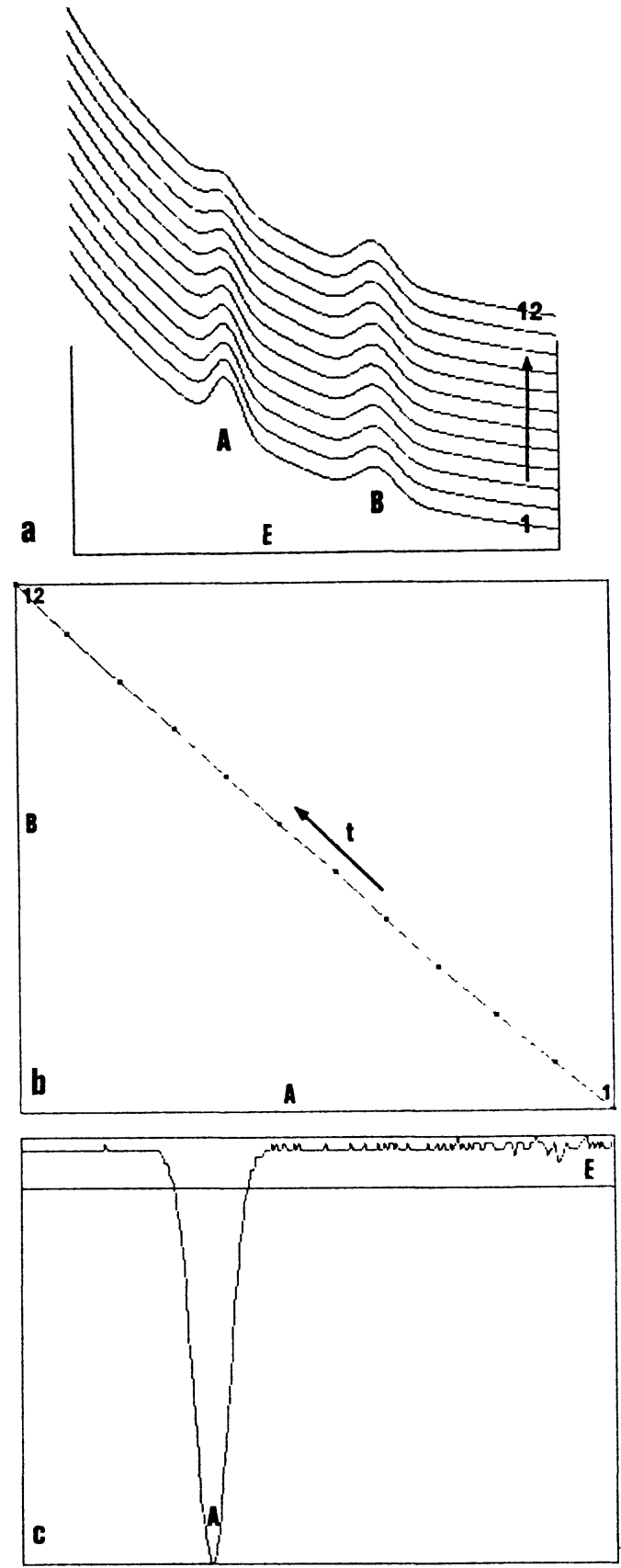

Fig. 4. - a) Time series of 12 other simulated spectra. In these spectra, the content of element $A$ is supposed to decrease as in figure 3a. The content of element B is supposed to be constant, but the incident beam current is supposed to increase continuously along the series. b) Scatter diagram showing the content of an energy window around the characteristic peak of element B, as a function of the content of an energy window for element $\mathrm{A}$. There is an apparent anti-correlation between the two evolutions. c) Multivariate Statistical Analysis (Correspondence Analysis) applied to the whole data set shows that only the window A contains some useful information. The evolution in window B is similar to the evolution of the background and therefore does not contain any significant information. 

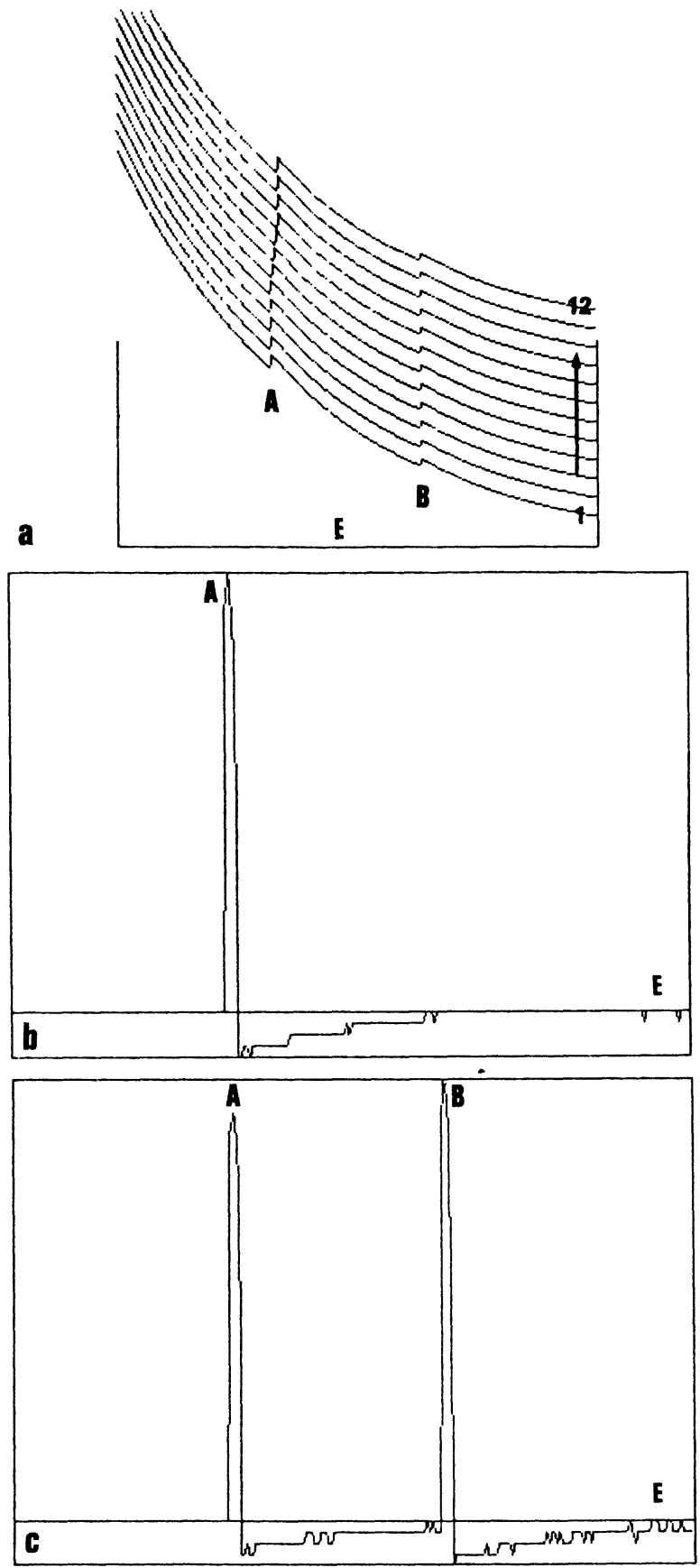

Fig. 5. - a) Time series of 12 simulated spectra. The spectra contain two characteristic edges A and B. Edge $\mathrm{B}$ is at constant position. Edge $\mathrm{A}$ is at constant energy position from spectrum 1 to spectrum 5 and then moves continuously from spectrum 6 to spectrum 12 (one energy channel drift per spectrum). b) This "chemical" drift is shown on the plot of the "channels of importance" on the factorial axis number 1. c) In another series we simulated an instrumental drift which resulted in a shift of the whole spectrum (one energy channel per spectrum). In that case, all the characteristic edges in the data set contribute to the "channels of importance", on axis 1. 

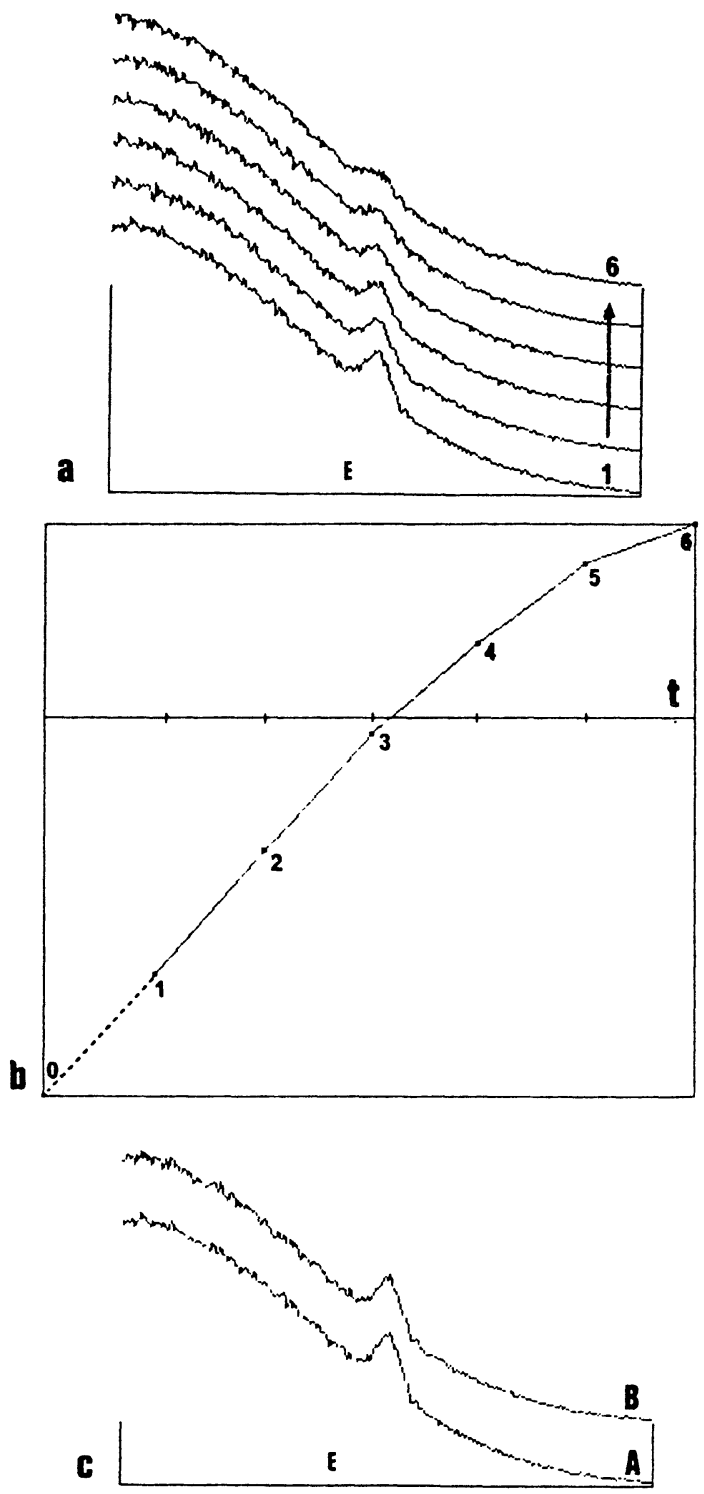

Fig. 6. - Extrapolation to zero-dose. a) Time series of 6 noisy simulated spectra. The content of the analyzed specimen in a given element is supposed to decrease according to a $\mathrm{e}^{-\alpha t}$ law. b) Only one factorial axis is important (the other ones contain mainly noise). The coordinates of the spectra on the factorial axis 1 follow a continuous curve as a function if time. This evolution can be estimated and then, provided some assumptions can be made, an extrapolation to zero-time (i.e. to zero-dose) can be performed, giving the weight of hypothetical spectrum number 0 on the factorial axis 1 . c) With this extrapolated coordinates, it is possible to reconstitute a spectrum at zero-dose (display A). This reconstitution compares very favourably with the simulation for $t=0$ (display B). 
to perform extrapolation. With this hypothesis, one is able to fit the weights of spectra $n=1$ to $n=6$ on axis 1 and then to deduce the weight of spectrum number 0 on this axis. With this weight, it is possible to reconstitute spectrum number 0 on the basis of the eigenvectors, resulting from the MSA of the experimental spectra. The result is shown in figure $6 \mathrm{c}$ and is compared to the "true" spectrum, which is known here since we are working with a simulation. There is a very good agreement between the amplitude values of the two peaks. One can even notice that the "invented" spectrum is less noisy than the "true" spectrum. This is due to the fact that, during the course of the synthesis, factorial axes 2 to 5 have been discarded and since they contain most of the noise of the experimental series, noise filtering has been performed at the same time.

\section{Application to an experimental situation.}

We submitted to MSA the experimental data set described in section 2, that is the X-ray spectra emitted by a glass as a function of the irradiation time [2]. In a classical analysis, it is necessary to define one or two energy windows of interest. The outcome of such an analysis (scatter diagram method for instance) is that there is an anticorrelation between the evolution of $\mathrm{Na}$ and that of Si.

We submitted these data first to Correspondence Analysis, a variant of MSA in which lines and columns of the data matrix $X_{i j}$ are normalized.

As a first result, we obtain the normalized eigenvalues in the matrix diagonalization:

$$
\frac{\lambda_{\alpha}}{\sum_{\alpha} \lambda_{\alpha}}=81 \% ; 7 \% \ldots \quad \text { for axes } 1,2, \ldots
$$

indicating that the evolutions throughout the series are not random but obey some deterministic rules.

The weights of the spectrum numbers on the factorial axis 1 are displayed in figure $7 \mathrm{a}$ and again one can see that there is some monotonous evolution from spectrum number 1 to spectrum number 23.

The weights of the energy channels on axis 1 are displayed on figure $7 \mathrm{~b}$. The strong (absolute) values of these weights indicate which are the channels which strongly contribute to the explanation of axis 1, that is, to the main variance (and therefore the main information) in the data set. One can see that the Na contribution is the most important, followed by the contributions of $\mathrm{Si}$ and $\mathrm{Au}$. We also see that $\mathrm{Si}$ and $\mathrm{Na}$ have anticorrelated evolutions (different signs). The relative contribution of $\mathrm{Si}$, compared to that of $\mathrm{Na}$, seems less important than expected from the scatter diagram representation (Fig. 1b).

It should be stressed that all these results have been obtained automatically, i.e., without any user intervention concerning the energy windows of interest.

In order to understand the discrepancy between results obtained with the scatter diagram representation and the Correspondence Analysis, we worked out another variant of MSA, i.e. Principal Component Analysis (PCA), in which the data matrix is not normalized at the initial step of the computation. The weights of the energy channels on axis 1 (which carries in this case $72 \%$ of the total variance) are displayed in figure $7 \mathrm{c}$. In that case, we find that the importance of Si (relative to $\mathrm{Na}$ ) is greater than when using $\mathrm{CA}$ and the result seems closer to the result obtained with the scatter diagram. This can be understood by the fact that CA studies the relative evolutions of the energy channels $\left(I(E) / \sum_{E} I(E)\right)$, while PCA studies their absolute evolutions $(I(E))$. 

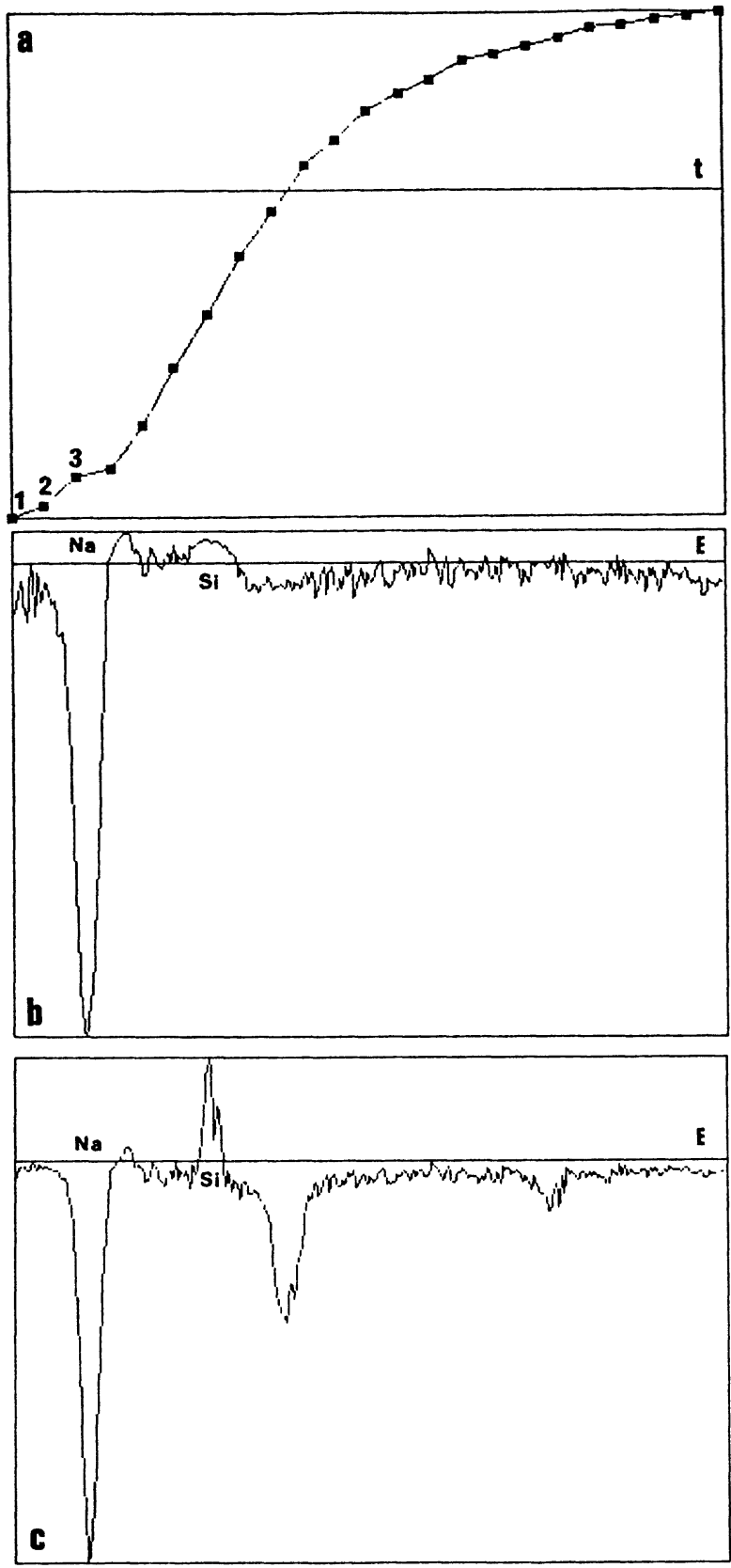

Fig. 7. - Multivariate Statistical Analysis applied to the experimental EDX series displayed in figure 1a. a) Weight of the experimental spectra on the factorial axis 1, showing a monotonous evolution as a function of time. b) Weight of the energy channels on axis 1, obtained by Correspondence Analysis. In this representation, one can see that the relative evolution is much more important for $\mathrm{Na}$ than for Si. c) Equivalent result obtained by Principal Component Analysis. In terms of absolute evolution, the information in the Si peak is greater than in terms of relative evolution. 
In this specific application, if one looks carefully at the original data, one can see that the total signal $\sum_{E} I(E)$ increases as a function of time, so that there is an overestimation of the Si increase in the scatter diagram. Working with relative values (as does CA) would be more correct. CA does this work automatically.

\section{Conclusion.}

Gathering important volumes of data in order to investigate in more depth the content of a specimen is, of course, advisable. But analyzing the content of these data is not always easy. Some artefacts can be produced by sophisticated data acquisition procedures (current drift in time dependent spectroscopy, for instance).

Among the different specific tools which can be used to process large data set, Multivariate Statistical Analysis seems to be a good candidate.

Its first use is to analyse the data set:

- the weights of the spectra on the factorial axes (and its representation as a two-dimensional plot) are useful to visualize the proximity between spectra and the shape of some global evolution function (see Figs. 3b, 7a);

- the weights of the energy channels on the factorial axes are a direct indication of the channels which contribute to the significant information in the series, without any need to define an a priori energy window of interest (see Fig. 3c).

Its second use is to process the data set: once all the information is plotted into several orthogonal sources of information, one is able to reconstitute the data set after filtering, i.e. removing some undesired factorial components. This is specifically useful for rejecting noise components which are mainly concentrated in high order eigenvectors.

The third use is to interpolate or extrapolate the data set. From the decomposition into eigenvalues, it is possible to reconstitute a fictitious member of the series by setting fictitious weights to these eigenvalues. Of course, these weights must not be chosen randomly, but must be deduced from the original weights according to some hypotheses. This process can be applied to zero dose extrapolation in time dependent spectroscopy.

\section{Acknowledgements.}

The series of spectra displayed in figures 1 and 3 was obtained by O. Jbara, X. Thomas and J. Cazaux in the "Laboratoire d'Analyse des Solides, Surfaces et Interfaces" (LASSI). We acknowledge their permission to use it in this work. We would also like thank A. Quiqueret for secretarial assistance.

\section{References}

[1] Craven A., Cluckie M., Duckworth S. and Baker T., Ultramicroscopy 28 (1989) 330.

[2] Tencé M., Walls M., Jeanguillaume C., Colliex C., Thomas X., Jbara O. and CazauX J., Inst. Phys. Conf. Ser. 98 (1989) 311.

[3] Weiss J., ReZ P., Higgs A., DAS ChOMdhuRY K. and MC CARTNEY M. Proc. 47th EMSA meeting (San Francisco Press) 406. 
[4] Jeanguillaume C., Thesis, Orsay, France (1989).

[5] Jeanguillaume C., J. Microsc. Spectrosc. Electron. 10 (1985) 409.

[6] el Gomati M., Peacock D., PruTton M. and Walker C., J. Microsc. 147 (1987) 149.

[7] JBara O., Thomas X. and CAZAuX J., Proc. XII th International Congress on X-ray optics and microanalysis, Cracow (1989) 552.

[8] Pearson K., Philos. Mag. 2 (1901) 559.

[9] Spearman C., Amer. J. Psychol. 15 (1904) 72 and 201.

[10] BENZECRI J.P., Methodologies of pattern recognition (Watanabe ed: Academic Press, New York, 1969).

[11] BENZECRI J.P., L'analyse des données (Dunod, Paris 1978).

[12] BARBER D., DUTHIE H. and WARD A., Proc. Symp. Knoxville, IAEA, Vienne (1975) 185.

[13] di PaOla R., Bazin J.P., Aubry F., Aurengo A., Cavailloles F., Henry J. and Kahn E., IEEE Trans. Nucl. Sci. NS 2 (1982) 1310.

[14] Frank J. and van HeEl M., J. Mol. Biol. 161 (1982) 134.

[15] VAN HeEl M. and Frank J., Pattern Recognition in Practice (GELSEMA ES and KANAL LN Eds., North Holland Publishing Company, 1980) p. 235.

[16] VAN HEEL M. and FRANK J., Ultramicroscopy 6 (1981) 187.

[17] Prutton M., el Gomati M. and Walker C., Inst. Phys. Conf. Ser. 90 (1987) 1.

[18] PRUTTON M., EL GOMATI M. and KenNy P., J. Electron. Spectroscopy and Related Phenomena 52 (1990) 197.

[19] HANNEQUIN P. and BONNET N., Optik 81 (1988) 6.

[20] BONNET N. and HANNEQUIN P., Ultramicroscopy 28 (1989) 248.

[21] TREBBIA P. and BONNET N., Ultramicroscopy 34 (1990) 165.

[22] TREBBIA P. and MORY C., Ultramicroscopy 34 (1990) 179.

[23] LEBART L., MoRINEAU A. and FENELON J.P., Traitement des données statistiques (Dunod, Paris, 1982).

[24] BRETAUDIERE J.P. and FranK J., J. Microsc. 144 (1986) 1.

[25] Bonnet N. and HanneQuin P., Inst. Phys. Conf. Ser. 93 (1988) 183. 